\title{
Outcomes in a nurse-led peripherally inserted central catheter program: a retrospective cohort study
}

\author{
Sheryl McDiarmid RN MBA, Nicholas Scrivens BSc, Marc Carrier MD, Elham Sabri MSc, \\ Baldwin Toye MD, Lothar Huebsch MD, Dean Fergusson PhD
}

\section{Abstract}

Background: Peripherally inserted central catheters (PICCs) provide enormous benefit to patients. However, recent publications have highlighted relatively high PICC-associated complication rates. We report on patient and device outcomes from a nurse-led program.

Methods: We performed a retrospective analysis of a prospective cohort of consecutive patients undergoing PICC insertion at The Ottawa Hospital between Jan. 1, 2013 and Dec. 31, 2014. Of the 8314 BioFlo PASV PICCs inserted, we randomly selected a sample of 700 and obtained a complete data set for 656 . We measured the cumulative incidence of major complications (catheterrelated bloodstream infections and deep vein thrombosis) and use of a thrombolytic to alleviate occlusions.

Results: The total number of catheter days was 58 486, and the median dwell time 45 days. We observed 4 cases of catheterrelated bloodstream infection $(0.6 \%$ [95\% Cl $0.17 \%-1.55 \%])(0.07 / 1000$ catheter days). Ten patients $(1.5 \%$ [95\% $\mathrm{Cl} 0.83 \%-$ $2.78 \%])(0.17 / 1000$ catheter days) had catheter-related deep venous thrombosis. At least 1 dose of thrombolytic was required in 75 catheters $(11.4 \%$ [95\% Cl 8.61\%-13.39]), $31(7.1 \%)$ of the 436 single-lumen catheters and $113(25.7 \%)$ of the 440 lumina of dual-lumen catheters $(p<0.001)$.

Interpretation: We attribute our low rates of major complications to a nurse-led expert insertion team, standardized care and maintenance protocols, high insertion volumes, novel catheter material and continuous quality-improvement initiatives that are implemented and evaluated regularly. We conclude that the considerable benefits PICCs provide to patients are attained with a low risk of major complications.

$\mathrm{P}$ eripherally inserted central catheters (PICCs) have transformed the care of patients across a wide variety of clinical settings, from tertiary to ambulatory, by providing a safe and reliable alternative to short-term centrally placed venous catheters. PICCs, placed with ultrasound guidance into upper arm veins, have a high successful insertion rate and a low insertion-related complication rate, are cost effective and can be used for a wide variety of infusion therapies. ${ }^{1,2}$ Although prescribed primarily by physicians, the prevailing practice model involves insertion of PICCs by a team comprising specially trained registered nurses supported by a compendium of infusion therapy standards of practice. ${ }^{3}$ The ongoing care and maintenance of PICCs has always been the responsibility of the registered nurse. Previous studies have shown that the rate of complications is correlated with the knowledge and expertise of care providers throughout the dwell time of the catheter., ${ }^{4,5}$

Although PICCs provide obvious benefits to patients, recent publications have raised safety concerns. ${ }^{3,6,7}$ Systematic reviews and observational studies have suggested that PICCs may expose patients to an unacceptably high risk of venous thromboembolism and catheter-related bloodstream infections, causing angst among clinicians who prescribe PICCs and those responsible for their insertion and subsequent care and maintenance. We performed a retrospective study to determine the risk of PICC-related complications in a nurseled vascular access care program.

Competing interests: AngioDynamics partly funded this study through a research agreement administered by the Ottawa Hospital Research Institute. Sheryl McDiarmid has given invited lectures for AngioDynamics, Bard and Teleflex-Arrow. No other competing interests were declared.

This article has been peer reviewed.

Correspondence to: Sheryl McDiarmid, smcdiarmid@toh.on.ca CMAJ Open 2017. DOI:10.9778/cmajo.20170010 


\section{Methods}

\section{Design and setting}

We conducted a retrospective analysis of a prospective cohort study that included consecutive patients undergoing a PICC insertion at The Ottawa Hospital between Jan. 1, 2013 and Dec. 31, 2014. The Ottawa Hospital is one of North America's largest academic health sciences centres and has about 1122 inpatient beds, roughly 50000 admissions annually and over 1.5 million outpatient visits annually. This includes the University of Ottawa Heart Institute and The Ottawa Hospital Regional Cancer and Rehabilitation programs. ${ }^{8}$ The Central Vascular Access Program is led by an advanced practice nurse who is accountable for oversight of the insertion, care and maintenance of all nondialysis central venous catheters in both inpatient and outpatient populations. As part of the program, data on all PICC insertions are collected prospectively.9

All PICCs were placed by experienced registered nurses, each performing 400-500 PICC insertions annually. The Safer Healthcare Now! central line insertion bundle, ${ }^{10}$ which consists of hand hygiene, barrier precautions and chlorhexidine skin antisepsis, was adhered to. Optimal catheter size (single- or dual-lumen) was determined by adequate vein diameter. Care and maintenance protocols based on the Infusion Nurses Society Infusion Therapy Standards of Practice ${ }^{11}$ were developed in conjunction with clinical nurse educators from The Ottawa Hospital and community providers. The Ottawa Hospital vascular access team is responsible for instilling a thrombolytic to treat occluded PICC lumens. No thrombolytics were administered in the community.

All catheters inserted were 4 French single-lumen or 5 French double-lumen BioFlo PASV PICCs (AngioDynamics, Inc). ${ }^{12}$ Ultrasound technology was used for all catheter insertions. Catheter tip placement was confirmed by routine chest radiography.

All PICCs were secured with a standard Tegaderm I.V. Advanced Securement Dressing (3M). No antimicrobial device was placed at the site at insertion or throughout the catheter dwell time. Three needleless connector devices were used during the study period: the One-Link or Interlink (Baxter Healthcare Corporation) for inpatients, and the MaxPlus (CareFusion, now a Becton, Dickinson and Company company) for community care providers. Catheter occlusions that could not be alleviated were treated with a tissue plasminogen activator, Cathflo (Roche), as per a predefined protocol. All catheters were routinely flushed with $10 \mathrm{~mL}$ of $0.9 \%$ sodium chloride (PosiFlush XS prefilled syringe [BD]). No heparin products were used to flush or lock the PICC.

Patients were followed from PICC insertion until removal.

\section{Outcomes}

The primary outcomes of the study were catheter-related bloodstream infection and symptomatic catheter-related deep venous thrombosis. We defined catheter-related bloodstream infection as the presence of bacteremia originating from the PICC according to the definition established by the Centers for Disease Control and Prevention, ${ }^{13}$ which includes a posi- tive blood culture result from the catheter and/or from a peripheral vein together with clear evidence that the catheter was the source; in addition, the patient had to manifest clinical symptoms of an infection. We defined catheter-related deep venous thrombosis as an occlusive or nonocclusive filling defect in the deep veins (brachial, axillary or subclavian) on the ipsilateral side proximal to the PICC insertion site detected on ultrasonography or venography. Testing was performed only if patients presented with clinical symptoms. Secondary outcome measures included superficial vein thrombosis (defined as a filling defect within the cephalic, basilic, median cubital or accessory cephalic vein on ultrasonography or venography) and catheter occlusion (defined as an obstruction of the catheter lumen that prevents or limits the ability to flush, withdraw blood, and/or administer solutions or medications). The thrombolytic dosage was captured per lumen, not per device. All outcome events were adjudicated by 2 physicians (B.T. and M.C.).

\section{Sample size and statistical analysis}

Of the 8314 PICCs inserted during the prospective study period, we selected a random sample of 700 using the randomnumber generator function of Microsoft Excel. Sample size was derived to ensure precise confidence intervals (CIs) around an expected event rate of our primary outcome (catheterrelated bloodstream infection or deep venous thrombosis). A sample size of 700 produces a two-sided $95 \%$ CI with a width equal to $3 \%$. The data were supplemented and verified with the electronic medical record of the 700 patients.

We calculated a descriptive summary of the baseline characteristics along with proportions and $95 \%$ CIs for all primary and secondary outcomes using the Wilson method without continuity correction. All statistical calculations were conducted with the use of StatsDirect version 2.8.0 (StatsDirect Ltd.).

\section{Ethics approval}

This study was approved by The Ottawa Hospital Research Ethics Board.

\section{Results}

Of the 700 patients, 44 were excluded because the PICC removal date was not available $(n=7)$ or the patient was transferred to another facility $(n=37)$. Thus, the data for 656 patients were analyzed. Baseline characteristics of the study population, ordering service and reason for insertion are given in Table 1 . Table 2 describes catheterization characteristics. The cumulative dwell time was 58486 (median 45) catheter days (range 1-842 catheter days).

We observed 4 cases of catheter-related bloodstream infection $(0.6 \%$ [ $95 \%$ CI $0.17 \%-1.55 \%])(0.07 / 1000$ catheter days). The organisms cultured were Escherichia coli, Enterococcus, coagulase-negative Stapbylococcus and, in 1 case, both S. aureus and group B Streptococcus. The time to infection after PICC insertion was 14-112 days. Three infections occurred in patients with hematological malignant disorders 
and 1 in a known intravenous drug user. All infections occurred in the inpatient setting.

Ten patients (1.5\% [95\% CI 0.83\%-2.78\%]) (0.17/ 1000 catheter days) had catheter-related deep venous thrombosis, of the upper extremity in all cases, during the follow-up period (Table 3 ). An additional 4 patients had superficial vein thrombosis, for a total of 14 patients $(2.1 \%$, 95\% CI $1.27 \%-$ $3.55 \%$ ) with thrombotic complications. Incidental findings of pulmonary embolism with negative findings on lower- and upper-extremity ultrasonography were detected in 2 additional patients.

Both catheter-related bloodstream infections (3/4 [75\%]) and catheter-related deep venous thrombosis (3/10 [30\%]) were more common in patients with hematological malignant disorders than in patients with other disorders. The incidence of deep venous thrombosis in this subgroup was $0.4 / 1000$ catheter days, compared to $0.17 / 1000$ catheter days in the overall cohort.

A thrombolytic was required in 75 cases $(11.4 \%$ [95\% CI $8.61 \%-13.39 \%])$. Of the 144 doses given, 31 (21.5\%) were administered into single-lumen catheters $(n=436)$, and 113 (78.5\%) were administered into a lumen of a double-lumen catheter $(n=220)$. When analyzed by lumen, $31(7.1 \%)$ of the 436 single-lumen catheters required a thrombolytic, compared to $113(25.7 \%)$ of the 440 lumina of the double-lumen catheters $(p<0.001)$ (Table 4).

\section{Interpretation}

We found low rates of catheter-related bloodstream infection, catheter-related deep venous thrombosis and catheter occlusion necessitating a thrombolytic when PICC insertion and

Table 1: Baseline characteristics of 656 patients with peripherally inserted central catheters at The Ottawa Hospital

\begin{tabular}{|lc|}
\hline Characteristic & No. (\%) of patients* \\
\hline Age, mean \pm SD (range), yr & $62 \pm 15(17-97)$ \\
\hline Female sex & $329(50.2)$ \\
\hline Ordering service & $217(33.1)$ \\
\hline Medicine & $186(28.4)$ \\
\hline Oncology & $128(19.5)$ \\
\hline Surgery & $63(9.6)$ \\
\hline Malignant hematology & $39(5.9)$ \\
\hline Cardiology & $23(3.5)$ \\
\hline Intensive care unit & $341(52)$ \\
\hline Reason for insertion & $229(35)$ \\
\hline Antibiotics & $37(6)$ \\
\hline Chemotherapy & $49(7)$ \\
\hline Parenteral nutrition & \\
\hline Other & \\
\hline $\begin{array}{l}\text { Note: SD = standard deviation. } \\
{ }^{*} \text { Except where noted otherwise. }\end{array}$ & \\
\hline
\end{tabular}

subsequent care and maintenance were performed by an expert nursing team. These findings are reassuring given the widespread use of PICCs.

\begin{tabular}{|lc|}
\hline \multicolumn{2}{|l|}{ Table 2: Catheterization characteristics } \\
\hline Characteristic & $\begin{array}{c}\text { No. }(\%) \text { of patients } \\
n=656\end{array}$ \\
\hline Right-sided insertion & $551(84.0)$ \\
\hline Vein cannulated & $512(78.0)$ \\
\hline Basilic & $131(20.0)$ \\
\hline Brachial & $13(2.0)$ \\
\hline Cephalic & $220(33.5)$ \\
\hline Catheter type & $436(66.5)$ \\
\hline Double-lumen & $603(91.9)$ \\
\hline Single-lumen & $53(8.1)$ \\
\hline No. of insertion attempts & \\
\hline 1 & $590(89.9)$ \\
\hline 2 & $66(10.1)$ \\
\hline $\begin{array}{l}\text { Catheter tip location } \\
\text { Cavoatrial junction/distal superior } \\
\text { vena cava }\end{array}$ & $58486(45)$ \\
\hline Mid superior vena cava & \\
\hline $\begin{array}{l}\text { Cumulative dwell time (median) } \\
\text { (range), catheter d }\end{array}$ & \\
\hline *Except where noted otherwise. & \\
\hline
\end{tabular}

Table 3: Characteristics of 10 patients with catheter-related deep vein thrombosis of the upper extremity

\begin{tabular}{|lc|}
\hline Characteristic & No. of patients* \\
\hline Male sex & 6 \\
\hline Time to thrombosis, median (range), d & 30 (4-259) \\
\hline Site & 6 \\
\hline Subclavian vein & 3 \\
\hline Axillary vein & 1 \\
\hline Brachial vein & \\
\hline Patient diagnosis & 3 \\
\hline Hematological malignant disorder & 2 \\
\hline Lung cancer & 1 \\
\hline Breast cancer & 1 \\
\hline Colorectal cancer & 3 \\
\hline Infection & 7 \\
\hline Outcome & 2 \\
\hline Complete resolution after first follow-up & 1 \\
\hline Extension of thrombosis & \\
\hline Residual nonocclusive thrombosis & \\
\hline *Except where noted otherwise. & \\
\hline
\end{tabular}




\section{OPEN}

Table 4: Thrombolytic use in 75 occluded catheters

\begin{tabular}{|lc|}
\hline Variable & $\begin{array}{c}\text { No. }(\%) \text { of } \\
\text { catheters } \\
n=656\end{array}$ \\
\hline No. of doses \\
\hline 1 & $38(5.8)$ \\
\hline 2 & $20(3.0)$ \\
\hline 3 & $7(1.1)$ \\
\hline$\geq 4$ & $10(1.5)$ \\
\hline Type of catheter & 31 lumina $(7.1)$ \\
\hline Single-lumen $(n=436)$ & 113 lumina $(25.7) \dagger$ \\
\hline \multicolumn{2}{|l|}{ Double-lumen $(n=220=440$ lumina) } \\
\hline $\begin{array}{l}* \text { Except where noted otherwise. } \\
\dagger p<0.001 .\end{array}$ \\
\hline
\end{tabular}

Our low rate of catheter-related bloodstream infection is consistent with previous reports. Maki and colleagues ${ }^{14}$ conducted a systematic review of 200 prospective studies that combined inpatients and outpatients; they reported a rate of infectious complications with various types of vascular access devices of 1.1/1000 catheter days. In contrast, Chopra and colleagues ${ }^{15}$ carried out a systematic review and meta-analysis of the risk of bloodstream infections associated with PICCs as compared to central venous catheters and reported a rate of $5.2 \%(76 / 1473)$ for inpatients and $0.45 \%$ (117/25 822) for outpatients. In a study of similar design to ours, Bouzad and colleagues ${ }^{16}$ reported an incidence of catheter-related bloodstream infections of 27 episodes, or 1.43/1000 catheter days, in a sample of 923 PICC placements; in the subcohort of patients with hematological disease, the overall rate of infectious complications was 3.13/1000 catheter days.

An important difference between our study and published evidence is the inclusion of patients as they transitioned from the acute care setting to the community. The median dwell time in our cohort was 45 catheter days. Although evidence supports that longer dwell times are associated with higher rates of catheter-related bloodstream infection, ${ }^{17}$ we did not observe this in our study. McLaws and Berry ${ }^{17}$ found overall incidence rates of catheter-related bloodstream infection with central venous catheters of $2.1,4.5$ and 10.2 cases/1000 catheter days during dwell intervals of 1-5 days, 6-15 days and 16-30 days, respectively.

In our study, 3 of the 4 (0.4/1000 catheter days) catheterrelated bloodstream infections occurred in patients with hematological malignant disorders, a population known to be at higher risk for this complication. This rate is considerably lower than that in previous reports. A literature review by Chopra and colleagues ${ }^{3}$ showed higher rates of PICC-related bloodstream infections among adult patients with cancer than among those without cancer $(1.1 / 1000$ catheter days v. 1.8$7.7 / 1000$ catheter days). Morano and colleagues ${ }^{18}$ reported on 612 patients with hematological disease with primarily $(86.8 \%$ of patients) 4 French single-lumen distally valved silicone
PICCs (Groshong [Bard Peripheral Vascular]); the incidence of catheter-related bloodstream infection was $7.7 \%$, or $0.59 /$ 1000 catheter days, and the mean dwell time was 101 days.

The rate of symptomatic deep venous thrombosis in our cohort was lower than that reported in the literature. In a recent meta-analysis, a subgroup analysis including inpatients and outpatients ( 8 studies, $n=9462$ ) gave a weighted frequency of PICC-related deep venous thrombosis of $3.4 \%$ (95\% CI $1.7 \%-5.19 \%)$ and an unweighted frequency of $3.0 \%$ (281/9462). ${ }^{7}$ The unweighted frequency of PICC-related deep venous thrombosis was $10.5 \%$ among patients in the intensive care unit ( 8 studies, $n=1219){ }^{7}$

In our study, catheter-related deep venous thrombosis was more prevalent in patients with hematological malignant disorders than in patients with other disorders. Although Chopra and colleagues ${ }^{3}$ reported an incidence of catheter-related thrombosis of $2 \%-5.5 \%$ among patients with diseases other than cancer, the incidence among those with cancer was $3.4 \%-7.8 \%$. In a previous study, we reported an incidence of catheter-related deep venous thrombosis of $5.6 \%$ (95\% CI $3.5 \%-8.6 \%$ ) in 340 patients with cancer (4 French singlelumen or 5 French double-lumen Groshong catheter)..$^{19}$ In a retrospective study of 899 PICCs placed in patients with hematological malignant disorders, Tran and colleagues ${ }^{20}$ reported a rate of symptomatic catheter-related deep venous thrombosis of $7.8 \%$. Although the catheter type was not available in $36 \%$ of cases, the prevalence of the complication varied by catheter type, from a low of $5.8 \%$ to a high of $11.8 \%$, which suggests that the catheter material may contribute to thrombosis. It is possible that the combination of preinsertion assessment of the patient and the vein by skilled registered nurses, a high rate of success on first insertion attempt and insertion of a maximum 5 French double-lumen PICC composed of a material that has been shown in in vitro testing to reduce thrombus accumulation ${ }^{12}$ contributed to our low rates of catheter-related deep venous thrombosis.

Catheter occlusion is a common and costly complication that diminishes the functionality of the PICC and interferes with timely administration of treatments. Rates of catheter occlusion of $14 \%-36 \%$ have been reported. ${ }^{21,22}$ In our study, $11 \%$ of PICC lumina required instillation of a thrombolytic. Strategies to prevent catheter occlusion - primarily adequate flushing protocols and catheter-appropriate needleless connectors - have been incorporated into the care and maintenance policies and procedures for The Ottawa Hospital and community care providers.

\section{Limitations and strengths}

Our study had a retrospective, single-centre, single-arm design, which limits the generalizability of the findings. The diagnosis of catheter-related bloodstream infection is often controversial, and infectious disease specialists disagree about the categorization of these infections. ${ }^{23} \mathrm{We}$ attempted to minimize bias with adjudication of events. Furthermore, although a multifaceted approach to reducing complications, including infection prevention practices, a dedicated team of expert registered nurses providing continuity of catheter care, and use of ultrasonography 
and novel catheter material, may have led to a reduced rate of catheter-associated complications, we are uncertain what role each of these components played in the overall outcome. The study did not have a control group, and therefore comparisons were not possible. Although we have attempted to compare complication rates relative to our study design, settings and methods, no published study to date encompasses a program structure comparable to that at The Ottawa Hospital.

Our study also has important strengths, including the large number of catheter insertions, with prolonged dwell time. Furthermore, both inpatients and outpatients were included, and care protocols were standardized across care settings. Although our study was retrospective, the data collection was done concurrently as events occurred. In addition, inclusion of inpatients and outpatients from different services, including medical, surgical, critical care and hematology/oncology, makes the data set more generalizable to the real-world setting.

\section{Conclusion}

We report a composite of rates of serious PICC-related complications that are lower than those previously reported. We attribute our low rates to an experienced nurse-led team, high insertion volumes, standardized protocols for care and maintenance, novel catheter material and continuous quality-improvement initiatives that are implemented and evaluated regularly. We conclude that the considerable benefits PICCs provide to patients are attained with a low risk of major complications.

\section{References}

1. Moureau N. Vascular safety: it's all about PICCs. Nurs Manage 2006;37:22-7.

2. Ng PK, Ault MJ, Ellrodt AG, et al. Peripherally inserted central catheters in general medicine. Mayo Clin Proc 1997;72:225-33.

3. Chopra V, Anand S, Krein SL, et al. Bloodstream infection, venous thrombosis, and peripherally inserted central catheters: reappraising the evidence. $A m \mathcal{F}$ Med 2012;125:733-41.

4. Guerin K, Wagner J, Reins K, et al. Reduction in central line-associated bloodstream infections by implementation of a postinsertion care bundle. Am 7 Infect Control 2010;38:430-3.

5. Timsit JF, Bouadma L, Ruckly S, et al. Dressing disruption is a major risk factor for catheter-related infections. Crit Care Med 2012;40:1707-14.

6. Chopra V, Ratz D, Kuhn L, et al. Peripherally inserted central catheter-related deep vein thrombosis: contemporary patterns and predictors. 7 Thromb Haemost 2014; $12: 847-54$

7. Chopra V, Anand S, Hickner A, et al. Risk of venous thromboembolism associated with peripherally inserted central catheters: a systematic review and metaanalysis. Lancet 2013;382:311-25.

8. The Ottawa Hospital annual report 2015-2016. Fast facts. Ottawa: The Ottawa Hospital; 2016. Available: https://www.ottawahospital.on.ca/annualreport/fast -facts_en.html (accessed 2017 June 21).

9. McDiarmid S. Data: the real d in decision making. 7 Can Vasc Access Assoc 2012; 6(2):21-5.

10. Prevent central line infections: getting started kit. Ottawa: Safer Healthcare Now!, Canadian Patient Safety Institute; 2012. Available: www.patientsafetyinstitute.ca/ en/toolsResources/Documents/Interventions/Central\%20Line-Associated\%20 Bloodstream\%20Infection/CLI\%20Getting\%20Started\%20Kit.pdf (accessed 2017 June 21).
11. Infusion Nurses Society. Infusion therapy standards of practice. 7 Infus Nurs 2016;39:S1-114.

12. BioFlo PICC: making a material difference. Latham (NY): AngioDynamics. Available: www.angiodynamics.com/products/bioflopicc (accessed 2016 July 17).

13. O'Grady NP, Alexander M, Burns LA, et al. Guidelines for the prevention of intravascular catheter-related infections. Clin Infect Disease 2011;52:e162-93.

14. Maki DG, Kluger DM, Crnich CJ. The risk of bloodstream infection in adults with different intravascular devices: a systematic review of 200 published prospective studies. Mayo Clin Proc 2006;81:1159-71.

15. Chopra V, O'Horo J, Rogers A, et al. The risk of bloodstream infection associated with peripherally inserted central catheters compared with central venous catheters in adults: a systematic review and meta-analysis. Infect Control Hosp Epidemiol 2013;34:908-18.

16. Bouzad C, Duron S, Bousquet A, et al. Peripherally inserted central catheterrelated infections in a cohort of hospitalized adult patients. Cardiovasc Intervent Radiol 2016;39:385-93.

17. McLaws ML, Berry G. Nonuniform risk of bloodstream infection with increasing central venous catheter-days. Infect Control Hosp Epidemiol 2005;26: 715-9.

18. Morano SG, Latagliata R, Girmenia C, et al. Catheter-associated bloodstream infections and thrombotic risk in hematologic patients with peripherally inserted central catheters (PICC). Support Care Cancer 2015;23:3289-95.

19. Aw A, Carrier M, Koczerginski J, et al. Incidence and predictive factors of symptomatic thrombosis related to peripherally inserted central catheters in chemotherapy patients. Thromb Res 2012;130:323-6.

20. Tran H, Arellano M, Chamsuddin A, et al. Deep venous thrombosis in patient with hematological malignancies after peripherally inserted central venous catheters. Leuk Lymphoma 2010;51:1473-7.

21. Baskin JL, Pui CH, Reiss U, et al. Management of occlusion and thrombosis associated with long-term indwelling central venous catheters. Lancet 2009;374. 159-69.

22. The occlusion management guideline. Hamilton (ON): Canadian Vascular Access Association; 2013.

23. Beekmann SE, Diekema DJ, Huskins C, et al. Diagnosing and reporting of central line-associated bloodstream infections. Infect Control Hosp Epidemiol 2012;33:875-82.

Affiliations: The Ottawa Hospital (McDiarmid), Ottawa Hospital Research Institute (McDiarmid, Scrivens, Sabri); Department of Medicine (Carrier), Ottawa Hospital Research Institute at the University of Ottawa; Division of Microbiology and Infectious Diseases (Toye), University of Ottawa, The Ottawa Hospital; Department of Medicine (Huebsch), University of Ottawa, The Ottawa Hospital; Clinical Epidemiology Program (Fergusson), Ottawa Hospital Research Institute; Department of Medicine (Fergusson), University of Ottawa, Ottawa, Ont.

Contributors: Sheryl McDiarmid conceived and designed the study, provided oversight for data collection and analysis, and was the primary author of the manuscript. Nicholas Scrivens collated the data, and Elham Sabri analyzed the data. Lothar Huebsch assisted with data analysis and manuscript preparation. Dean Fergusson assisted with data analysis and interpretation, and manuscript preparation. Marc Carrier and Baldwin Toye adjudicated outcome events and assisted with manuscript preparation. All of the authors approved the final version to be published and agreed to act as guarantors of the work.

Funding: This study was partly funded by AngioDynamics through a research agreement administered by the Ottawa Hospital Research Institute.

Acknowledgement: The authors thank the Vascular Access registered nursing team at The Ottawa Hospital.

Supplemental information: For reviewer comments and the original submission of this manuscript, please see www.cmajopen.ca/content/5/3/ E535/suppl/DC1 